\title{
Aplicação do Método dos Mínimos Quadrados para o ajuste da curva de potência de um aerogerador de pequeno porte
}

\section{Application of the Least Squares Method to the adjusting the power curve of a small wind generator}

Camila e Silva Gomes Instituto Federal de Educação, Ciência e Tecnologia do Rio Grande do Sul (IFRS) Campus Rio Grande, Rio Grande, RS, Brasil Universidade Federal do Rio Grande (FURG), Centro Ciências Computacionais Programa de Pós Graduação em Modelagem Computacional (PPGMC), Rio Grande, RS, Brasil http://orcid.org/0000-0001-7078-4048, camila.gomes@riogrande.ifrs.edu.br

\author{
Nisia Krusche \\ Universidade Federal do Rio Grande (FURG), Centro Ciências Computacionais \\ Rio Grande, RS, Brasil \\ https://orcid.org/0000-0001-9880-4987, nkrusche@furg.br
}

Javier Garcia López

Instituto Federal de Educação, Ciência e Tecnologia do Rio Grande do Sul (IFRS)

Campus Rio Grande, Rio Grande, RS, Brasil http://orcid.org/0000-0002-9595-5568, javier.garcia@riogrande.ifrs.edu.br

\section{Informações do Artigo \\ Como citar este artigo \\ GOMES, Camila e Silva; KRUSCHE, Nisia; LÓPEZ, Javier Garcia. Aplicação do Método dos Mínimos Quadrados para o ajuste da curva de potência de um aerogerador de pequeno porte. REMAT: Revista Eletrônica da Matemática, Bento Gonçalves, RS, v. 6 , n. 1, p. 01-16, jan. 2020. DOI: https://doi.org/10.35819/remat2020v6i1id3492}

\section{Histórico do Artigo}

Submissão: 28 de maio de 2019.

Aceite: 12 de setembro de 2019.

\section{Palavras-chave}

Modelagem Matemática

Energia Eólica

Ajuste de Curvas

Método dos Mínimos Quadrados

Curva de Potência

\section{Resumo}

Com o desenvolvimento da indústria eólica e a necessidade de gerar energia elétrica, os aerogeradores estão sendo instalados em diversas localidades. Em cada ambiente de instalação são observadas certas peculiaridades, o que torna necessária uma modelagem adequada da relação entre a velocidade do vento e a potência gerada pelo aerogerador, a fim de prever corretamente a produção de energia em qualquer ambiente. Neste trabalho, foi construída uma proposta, a partir dos dados coletados por um aerogerador em junho de 2017, para encontrar uma representação polinomial para a curva de potência de um modelo de aerogerador específico, usando um método de ajuste de curvas. A representação obtida foi validada com os resultados diários coletados e com obtenção da curva de potência do aerogerador. Nos 14.929 dados registrados por minuto pelo aerogerador no mês, foi aplicado o Método de Ajuste por Mínimos Quadrados, utilizando um polinômio de grau 3 como base. O objetivo deste trabalho é encontrar um polinômio que represente de modo adequado a curva de potência de um aerogerador de pequeno porte. Uma vez encontrado esse polinômio, para auferir a qualidade da representação, foi avaliado o nível de correlação e significância, comparando-se o polinômio encontrado com dados experimentais coletados ao longo de 30 dias e a sua curva de potência. Os resultados obtidos mostram-se satisfatórios para os casos nos quais os experimentos permitiram coletar dados úteis por mais de 2 horas consecutivas.

\section{Abstract}

Keywords

Mathematical Modeling

Wind Energy

Curve Adjustment

Least Squares Methods

Power Curve
With the development of the wind industry and the need of generating electricity, wind turbines are being installed in several locations. In each installation environment certain peculiarities are observed, which do necessary to model the relationship between the wind speed and the power generated by the wind turbine, in order to predict correctly the energy production in any 
environment. In this paper, a proposal was made, based on data collected by a wind generator in June 2017 , to find a polynomial representation for the power curve of a specific wind turbine model, using a curve fitting method. The representation obtained was validated with the daily results collected and with the wind turbine power curve obtained which presented satisfactory results. In The 14929 data recorded per minute by the wind turbine during a month, the Least Squares Method was applied, using a grade 3 in these polynomial as base. The objective of this paper is to find a polynomial that adequately represents the power curve of a small wind turbine. Once this polynomial was found, the level of correlation and significance was evaluated to compare the polynomial found with experimental data collected over 30 days and the power curve of the polynomial. The results obtained are satisfactory for the cases in which the experiments allowed us to collect useful data for more than 2 consecutive hours.

\section{Introdução}

A eletricidade tem grande flexibilidade e facilidade de distribuição, além disso a demanda mundial está crescendo, impulsionada pelo crescimento no consumo por equipamentos eletrônicos e pela atividade industrial (BRASIL, 2007). O vento é uma fonte limpa e inesgotável de energia, atualmente utilizado também na geração da energia elétrica por meio dos aerogeradores. Com o desenvolvimento da indústria eólica, os aerogeradores estão sendo instalados em diversas localidades; em cada ambiente de instalação observam-se variações em relação à densidade do ar, à altitude e às condições climáticas sazonais, além da complexidade do terreno, o que influencia significativamente na produção de energia eólica local. Diante dessas peculiaridades, faz-se necessário dispor de uma modelagem adequada para a relação entre a velocidade do vento e a potência gerada pelo aerogerador (curva de potência), com a intenção de prever corretamente a produção de energia em qualquer ambiente.

Com o objetivo de prever a potência gerada por um aerogerador doméstico, utilizou-se o Método dos Mínimos Quadrados, método de ajuste de curva para gerar um polinômio que descreva corretamente a relação da potência em função da velocidade do vento deste aerogerador, para que seja possível fazer uma previsão da potência gerada por ele em qualquer ambiente adequado para sua instalação. A escolha do método dos mínimos quadrado é justificada pois as tentativas de representação usadas anteriormente, em particular a interpolação, com a qual nossos resultados serão comparados, se mostraram insuficientes.

\section{Referencial Teórico}

Em geral, a potência extraída do vento pelos aerogeradores (KASSEM; HÜSEYIN; ABDELRAHMAN, 2017) varia com o cubo da sua velocidade (Equação 1), o que reforça a importância da estimativa correta da velocidade do vento para a previsão de geração de energia.

$$
P=\frac{1}{2} \rho v^{3} A C_{p}
$$


onde, $P$ é a potência em Watts, $\rho$ a densidade do $\operatorname{ar}\left[\mathrm{kg} / \mathrm{m}^{3}\right], v$ a intensidade da velocidade do vento $[\mathrm{m} / \mathrm{s}], A$ a área varrida pelas pás do aerogerador $\left[\mathrm{m}^{2}\right]$ e $C_{p}$ coeficiente de potência que tem seu valor máximo igual a 0,59, também conhecido como limite de Betz (PINTO et al., 2018), no qual há a máxima potência extraída do vento na área de varredura da máquina. Com o desenvolvimento da indústria eólica, os aerogeradores estão sendo instalados em diversas localidades. Dessa forma, em cada ambiente de instalação observam-se variações em relação à densidade do ar, à altitude e às condições climáticas sazonais, além da complexidade do terreno, o que influencia significativamente na produção de energia local. Diante dessas peculiaridades, faz-se necessária uma modelagem adequada da relação entre a velocidade do vento e a potência gerada pelo aerogerador (curva de potência), com a intenção de prever corretamente a produção de energia em qualquer ambiente.

\subsection{Método dos Mínimos Quadrados}

O Método dos Mínimos Quadrados (LOPES, 2008) é usado para encontrar uma função $\mathrm{Y}_{\mathrm{k}}=\mathrm{F}\left(\mathrm{a}_{\mathrm{k}}, \mathrm{x}_{\mathrm{k}}\right)$, para $\left(\mathrm{x}_{\mathrm{k}}, \mathrm{y}_{\mathrm{k}}\right)$ conhecidos, de modo que usando os $\mathrm{X}_{\mathrm{k}}$, conseguimos calcular valores de $Y_{k}$, usando os $a_{k}$ calculados, e podemos compará-los com os $y_{k}$ conhecidos, de modo que a representação deixe o menor erro possível; quer dizer que $F\left(a_{k}, x_{k}\right)$ seja a melhor representação possível. Para tanto, construiremos uma função Erro e aplicaremos a Técnica de Máximos e Mínimos para funções de várias variáveis:

$$
\text { Erro }=\sum_{k=1}^{n}\left(y_{k}-Y_{k}\right)^{2}
$$

ou seja, a diferença entre o $y_{k}$ conhecido e o $Y_{k}$ calculado, usando a função $F\left(a_{k}, x_{k}\right)$. Dessa forma, as variáveis que determinam o erro são as variáveis $a_{k}$, podendo assim escrever a função Erro da seguinte maneira:

$$
\text { Erro }=\sum_{k=1}^{n}\left(y_{k}-F\left(a_{i}, x_{i}\right)\right)^{2}
$$

Logo, se calcularmos as derivadas parciais, correspondentes para cada $a_{i}$ e as igualarmos a 0, ter-se-á um sistema de equações da forma:

$$
\frac{\partial E r r o}{\partial a_{i}}=2 \sum_{k=1}^{n}\left(y_{k}-F\left(a_{i}, x_{k}\right)\right) \frac{\partial\left(F\left(a_{i}, x_{k}\right)\right)}{\partial a_{i}}=0
$$

A solução do sistema fornece a função de menor erro ou melhor representação. No Método dos Mínimos Quadrados para polinômios de grau $\mathrm{n}$, para generalizar este resultado ajustando qualquer polinômio da forma $y=a_{0}+a_{1} x+\ldots+a_{n} x^{n}$ aos pontos $\left(x_{i}, y_{i}\right)$, basta fazermos:

$$
r_{i}=y_{i}-\left(a_{0}+a_{1} x_{i}+a_{2} x_{i}^{2} \ldots+a_{n} x_{i}^{n}\right)
$$


Dessa forma, o erro é dado por:

$$
\sum r_{i}^{2}=\sum\left(y_{i}-\left(a_{0}+a_{1} x_{i}+a_{2} x_{i}^{2} \ldots+a_{n} x_{i}^{n}\right)\right)^{2}
$$

Então, para encontrarmos os coeficientes $a_{0}, a_{1}, \ldots, a_{n}$, é necessário resolver o mesmo sistema $A^{\top} A X=A^{\top} Y$. Efetuando os cálculos de $A^{\top} A$ e de $A^{\top} Y$, temos:

$$
\left(\begin{array}{cccccc}
\sum_{i=1}^{p} x_{i}^{0} & \sum_{i=1}^{p} x_{i}^{1} & \sum_{i=1}^{p} x_{i}^{2} & \ldots & \sum_{i=1}^{p} x_{i}^{n} \\
\sum_{i=1}^{p} x_{i}^{1} & \sum_{i=1}^{p} x_{i}^{2} & \sum_{i=1}^{p} x_{i}^{3} & \ldots & \sum_{i=1}^{p} x_{i}^{n+1} \\
\vdots & \vdots & \vdots & \cdots & \vdots \\
\sum_{i=1}^{p} x_{i}^{j} & \sum_{i=1}^{p} x_{i}^{1+j} & \sum_{i=1}^{p} x_{i}^{2+j} & \ldots & \sum_{i=1}^{p} x_{i}^{n+j} \\
\vdots & \vdots & \vdots & & \vdots \\
\sum_{i=1}^{p} x_{i}^{n} & \sum_{i=1}^{p} x_{i}^{n+1} & \sum_{i=1}^{p} x_{i}^{n+2} & \cdots & \sum_{i=1}^{p} x_{i}^{2 n}
\end{array}\right) \cdot\left(\begin{array}{c}
a_{0} \\
a_{1} \\
a_{2} \\
\vdots \\
a_{j} \\
\vdots \\
a_{n}
\end{array}\right)=\left(\begin{array}{c}
\sum_{i=1}^{p} x_{i}^{0} y_{i} \\
p \\
\sum_{i=1}^{p} x_{i}^{1} y_{i} \\
p \\
\sum_{i=1}^{p} x_{i}^{2} y_{i} \\
\vdots \\
\sum_{i=1}^{p} x_{i}^{j} y_{i} \\
\vdots \\
\sum_{i=1}^{p} x_{i}^{n} y_{i}
\end{array}\right)
$$

Esse procedimento pode ser generalizado para qualquer curva de ajuste da forma: $y \cong$ $a_{0} g_{0}(x)+\ldots+a_{n} g_{n}(x)$, desde que as funções $g_{k}(x)$ para $\mathrm{k}=0,1,2,3, \ldots, \mathrm{n}$ avaliadas nos pontos resultem em vetores linearmente independentes, condição necessária para que a matriz $A^{\top} A$ seja invertível, o que garante a solução do sistema gerado.

\subsection{Análise Estatística}

O coeficiente de correlação de Pearson (r) (DUFFIE; YANG; BOLLINGER, 1985) assume valores entre -1 e 1, e mede o grau da correlação linear entre duas variáveis quantitativas:

$$
r=\frac{\sum\left(x_{i}-\bar{x}\right)\left(y_{i}-\bar{y}\right)}{\sqrt{\left(\sum\left(x_{i}-\bar{x}\right)^{2}\right)\left(\sum\left(y_{i}-\bar{y}\right)^{2}\right)}}
$$

Sendo $\bar{x}$ a média dos valores de $x$ :

- $r=1$ significa uma correlação perfeita positiva entre as duas variáveis.

- $r=-1$ significa uma correlação negativa perfeita entre as duas variáveis. Isto é, se uma aumenta, a outra sempre diminui.

- $r=0$ significa que as duas variáveis não dependem linearmente uma da outra.

O erro absoluto relativo (RAE) é o erro absoluto total relativo ao erro obtido se a previsão fosse simplesmente a média dos valores reais. Matematicamente, o erro absoluto relativo de um modelo é avaliado pela equação: 


$$
R A E=\frac{\sum_{j=1}^{n}\left|P_{i j}-T_{j}\right|}{\sum_{j=1}^{n}\left|T_{j}-\bar{T}\right|}
$$

onde $\mathrm{P}_{i j}$ é o valor previsto pelo modelo i para registro j (de $\mathrm{n}$ registros), Tj é o valor esperado e $\bar{T}$ é dado pela equação:

$$
\bar{T}=\frac{1}{n} \sum_{j=1}^{n} T_{j}
$$

O erro médio absoluto (MAE) é o erro médio de previsão (DUFFIE; YANG; BOLLINGER, 1985), calculado pela equação:

$$
M A E=\frac{\sum_{j=1}^{n}\left|P_{j}-T_{j}\right|}{n}
$$

onde Pj é o valor previsto pelo modelo i, Tj é o valor esperado e $n$ é o número total de registros.

\section{Metodologia}

Para construir o polinômio associado à curva de potência, foram utilizados os dados de potência inseridos na rede, no mês de junho de 2017, pelo aerogerador fabricado por ZM Bombas, do tipo trifásico de imãs permanentes com acoplamento direto, de $12 \mathrm{~m}$ de altura, com 128,15 Kg, $3 \mathrm{~kW}$ de potência nominal, 3 pás, $3,4 \mathrm{~m}$ de diâmetro do rotor e controle passivo. O aerogerador pertence ao projeto FURG-UFSM intitulado "Laboratório para Ensaio de Pequenos Aerogeradores" e está instalado nas dependências da Universidade Federal de Rio Grande (FURG) no município de Rio Grande, estado do Rio Grande do Sul (GEPOC, 2017). Foi utilizado como base de cálculo um polinômio de terceiro grau, o qual é uma das soluções proposta por Trancoso, Domingos e Pestana (2006). Essa solução apresentou correlação de $88 \%$, para velocidades baixas do vento, mas não produz bons resultados quando as velocidades são superiores a $15 \mathrm{~m} / \mathrm{s}$. O aerogerador utilizado neste trabalho, para essas velocidades, atua em potência nominal (constante). A utilização desse grau no polinômio faz sentido com a Equação (1). Dessa forma, utilizamos o Método dos Mínimos Quadrados para ajustar essa curva.

Aplicando o Método dos Mínimos Quadrados para o polinômio $y(x)=a x^{3}+b x^{2}+c x+d$, temos os seguintes casos:

a. Caso 1 - Polinômio completo

$$
\text { Erro }=\sum_{k=1}^{n}\left(y_{k}-a x_{k}^{3}-b x_{k}^{2}-c x_{k}-d\right)^{2}
$$

Calculam-se as derivadas parciais em $a, b, c$ e $d$, obtendo-se:

$$
\begin{aligned}
& \frac{\partial E r r o}{\partial a}=-2\left[\sum_{k=1}^{n}\left(y_{k}-a x_{k}^{3}-b x_{k}^{2}-c x_{k}-d\right) x_{k}^{3}\right] \\
& \frac{\partial E r r o}{\partial b}=-2\left[\sum_{k=1}^{n}\left(y_{k}-a x_{k}^{3}-b x_{k}^{2}-c x_{k}-d\right) x_{k}^{2}\right]
\end{aligned}
$$




$$
\begin{gathered}
\frac{\partial \text { Erro }}{\partial c}=-2\left[\sum_{k=1}^{n}\left(y_{k}-a x_{k}^{3}-b x_{k}^{2}-c x_{k}-d\right) x_{k}\right] \\
\frac{\partial E r r o}{\partial d}=-2\left[\sum_{k=1}^{n}\left(y_{k}-a x_{k}^{3}-b x_{k}^{2}-c x_{k}-d\right)\right]
\end{gathered}
$$

Igualando a 0 para achar os valores máximos do erro temos o sistema:

$$
\begin{gathered}
a \sum_{k=1}^{n} x_{k}^{6}+b \sum_{k=1}^{n} x_{k}^{5}+c \sum_{k=1}^{n} x_{k}^{4}+d \sum_{k=1}^{n} x_{k}^{3}=\sum_{k=1}^{n} y_{k} x_{k}^{3} \\
a \sum_{k=1}^{n} x_{k}^{5}+b \sum_{k=1}^{n} x_{k}^{4}+c \sum_{k=1}^{n} x_{k}^{3}+d \sum_{k=1}^{n} x_{k}^{2}=\sum_{k=1}^{n} y_{k} x_{k}^{2} \\
a \sum_{k=1}^{n} x_{k}^{4}+b \sum_{k=1}^{n} x_{k}^{3}+c \sum_{k=1}^{n} x_{k}^{2}+d \sum_{k=1}^{n} x_{k}=\sum_{k=1}^{n} y_{k} x_{k} \\
a \sum_{k=1}^{n} x_{k}^{3}+b \sum_{k=1}^{n} x_{k}^{2}+c \sum_{k=1}^{n} x_{k}+d n=\sum_{k=1}^{n} y_{k}
\end{gathered}
$$

b. Caso 2 - Termo independente nulo $(d=0)$

$$
\text { Erro }=\sum_{k=1}^{n}\left(y_{k}-a x_{k}^{3}-b x_{k}^{2}-c x_{k}\right)^{2}
$$

Calculam-se as derivadas parciais em $a, b$ e $c$, obtendo-se:

$$
\begin{aligned}
& \frac{\partial E r r o}{\partial a}=-2\left[\sum_{k=1}^{n}\left(y_{k}-a x_{k}^{3}-b x_{k}^{2}-c x_{k}\right) x_{k}^{3}\right] \\
& \frac{\partial E r r o}{\partial b}=-2\left[\sum_{k=1}^{n}\left(y_{k}-a x_{k}^{3}-b x_{k}^{2}-c x_{k}\right) x_{k}^{2}\right] \\
& \frac{\partial E r r o}{\partial c}=-2\left[\sum_{k=1}^{n}\left(y_{k}-a x_{k}^{3}-b x_{k}^{2}-c x_{k}\right) x_{k}\right]
\end{aligned}
$$

Igualando a 0 para achar os valores máximos do erro temos o sistema:

$$
\begin{aligned}
& a \sum_{k=1}^{n} x_{k}^{6}+b \sum_{k=1}^{n} x_{k}^{5}+c \sum_{k=1}^{n} x_{k}^{4}=\sum_{k=1}^{n} y_{k} x_{k}^{3} \\
& a \sum_{k=1}^{n} x_{k}^{5}+b \sum_{k=1}^{n} x_{k}^{4}+c \sum_{k=1}^{n} x_{k}^{3}=\sum_{k=1}^{n} y_{k} x_{k}^{2} \\
& a \sum_{k=1}^{n} x_{k}^{4}+b \sum_{k=1}^{n} x_{k}^{3}+c \sum_{k=1}^{n} x_{k}^{2}=\sum_{k=1}^{n} y_{k} x_{k}
\end{aligned}
$$


C. Caso 3 - Polinômio incompleto $\operatorname{com} c=0$

$$
\text { Erro }=\sum_{k=1}^{n}\left(y_{k}-a x_{k}^{3}-b x_{k}^{2}-d\right)^{2}
$$

Calculam-se as derivadas parciais em $a, b$ e $d$, obtendo-se:

$$
\begin{aligned}
& \frac{\partial E r r o}{\partial a}=-2\left[\sum_{k=1}^{n}\left(y_{k}-a x_{k}^{3}-b x_{k}^{2}-d\right) x_{k}^{3}\right] \\
& \frac{\partial E r r o}{\partial b}=-2\left[\sum_{k=1}^{n}\left(y_{k}-a x_{k}^{3}-b x_{k}^{2}-d\right) x_{k}^{2}\right] \\
& \frac{\partial E r r o}{\partial d}=-2\left[\sum_{k=1}^{n}\left(y_{k}-a x_{k}^{3}-b x_{k}^{2}-d\right)\right]
\end{aligned}
$$

Igualando a 0 para achar os valores máximos do erro temos o sistema:

$$
\begin{gathered}
a \sum_{k=1}^{n} x_{k}^{6}+b \sum_{k=1}^{n} x_{k}^{5}+d \sum_{k=1}^{n} x_{k}^{3}=\sum_{k=1}^{n} y_{k} x_{k}^{3} \\
a \sum_{k=1}^{n} x_{k}^{5}+b \sum_{k=1}^{n} x_{k}^{4}+d \sum_{k=1}^{n} x_{k}^{2}=\sum_{k=1}^{n} y_{k} x_{k}^{2} \\
a \sum_{k=1}^{n} x_{k}^{3}+b \sum_{k=1}^{n} x_{k}^{2}+d n=\sum_{k=1}^{n} y_{k}
\end{gathered}
$$

d. Caso 4 - Polinômio incompleto com $b=0$

$$
\text { Erro }=\sum_{k=1}^{n}\left(y_{k}-a x_{k}^{3}-c x_{k}-d\right)^{2}
$$

Calculam-se as derivadas parciais em $a, c$ e $d$, obtendo-se:

$$
\begin{aligned}
& \frac{\partial E r r o}{\partial a}=-2\left[\sum_{k=1}^{n}\left(y_{k}-a x_{k}^{3}-c x_{k}-d\right) x_{k}^{3}\right] \\
& \frac{\partial E r r o}{\partial c}=-2\left[\sum_{k=1}^{n}\left(y_{k}-a x_{k}^{3}-c x_{k}-d\right) x_{k}\right] \\
& \frac{\partial E r r o}{\partial d}=-2\left[\sum_{k=1}^{n}\left(y_{k}-a x_{k}^{3}-c x_{k}-d\right)\right]
\end{aligned}
$$

Igualando a 0 , para achar os valores máximos, do erro temos o sistema:

$$
\begin{aligned}
& a \sum_{k=1}^{n} x_{k}^{6}+c \sum_{k=1}^{n} x_{k}^{4}+d \sum_{k=1}^{n} x_{k}^{3}=\sum_{k=1}^{n} y_{k} x^{3} \\
& a \sum_{k=1}^{n} x_{k}^{4}+c \sum_{k=1}^{n} x_{k}^{2}+d \sum_{k=1}^{n} x_{k}=\sum_{k=1}^{n} y_{k} x_{k}
\end{aligned}
$$




$$
a \sum_{k=1}^{n} x_{k}^{3}+c \sum_{k=1}^{n} x_{k}+d n=\sum_{k=1}^{n} y_{k}
$$

Ao ajustar um polinômio para $n$ pontos é possível testar o modelo para diversos tipos de experimentos. Isso não significa que em cada caso, necessariamente, usaremos a mesma representação, mas sim, que para cada experimento acharemos sua melhor representação, a partir dos dados coletados.

Em seguida foi realizada uma análise estatística por meio da correlação do erro absoluto relativo e do erro médio absoluto.

Utilizou-se para o ajuste polinomial o software Octave ${ }^{1}$, mais especificamente o comando polyfit (MATSUMOTO, 2001). Para utilização dessa função, são dados uma série de valores $(x, y)$ e o grau do polinômio $n$; o software retorna os coeficientes do polinômio que melhor aproxima a curva dada pela série em $(x, y)$. Esse software obteve o polinômio interpolador, o qual, ao compará-lo com nossos resultados, mostrou-se de menor qualidade de representação do fenômeno.

Os polinômios resultantes do nosso método foram comparados com a proposta do polinômio gerado pelo software matemático.

\section{Resultados e Discussão}

Os dados utilizados como base para a resolução dos sistemas de equações foram a intensidade da velocidade do vento (variável $x$ ) e a potência ativa injetada na rede (variável $y$ ), disponíveis no site GEPOC (2017). Dos dados originais coletados pelo aerogerador a cada minuto, no mês de junho de 2017, foram selecionados apenas aqueles obtidos nos instantes em que a velocidade do vento registrada era maior que $3 \mathrm{~m} / \mathrm{s}$, pois para velocidades inferiores não deveriam haver registros de energia, já que as pás do aerogerador não se movem, e naqueles em que havia registro de potência nula, já que em alguns instantes houve erros de registro de potência, mesmo com vento propício para a geração de energia. Restaram 14.929 dados válidos, os quais foram utilizados para a realização do ajuste de curva; mesmo passando por um filtro ainda restaram dados registrados fora da curva.

Após a determinação dos coeficientes por meio dos sistemas, encontrou-se os seguintes polinômios:

$$
\begin{gathered}
P 1=1,9876 \cdot v^{3}+1,23104026 \cdot v^{2}-6,374544095 \cdot v-0,000576451 \\
P 2=2,193703 \cdot v^{3}-1,09643 \cdot v^{2}+0,009893 \cdot v \\
P 3=2,193703 \cdot v^{3}-1,09643 \cdot v^{2}+0,009893 \\
P 4=2,047805 \cdot v^{3}+1,56032 \cdot v-16,2587
\end{gathered}
$$

\footnotetext{
${ }^{1}$ Disponível em: https://www.gnu.org/software/octave/. Acesso em: 27 dez. 2019.
} 
$\mathrm{Na}$ Figura 1, em azul (.), estão representados os dados originais registrados pelo aerogerador em junho de 2017; em preto (x) os instantes em que a intensidade do vento registrada foi maior que $3 \mathrm{~m} / \mathrm{s}$; e em vermelho (o) os dados finais utilizados no ajuste da curva.

Figura 1 - Seleção dos dados utilizados no ajuste de curva.

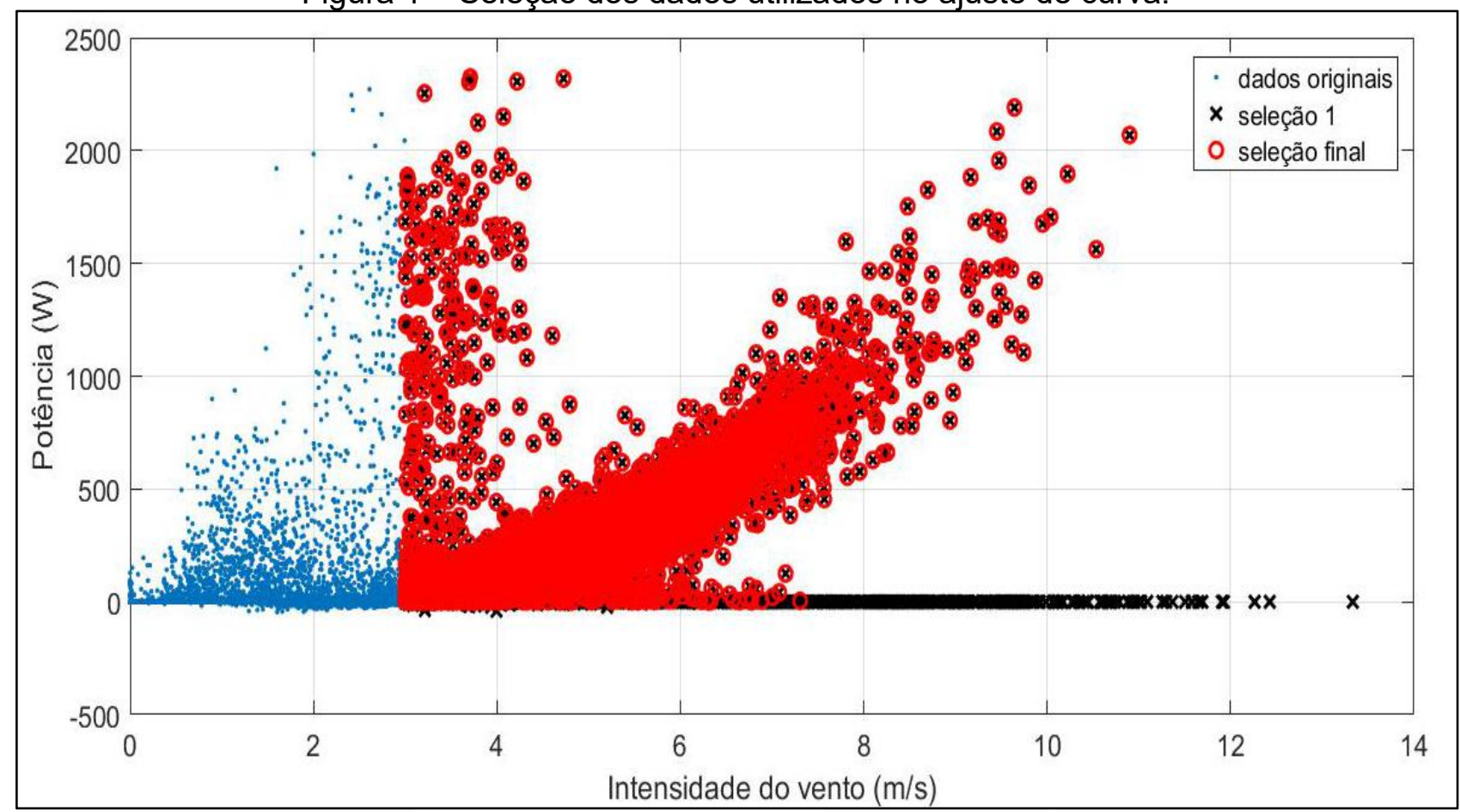

Fonte: Dados coletados em GEPOC (2017).

Para análise dos resultados, comparou-se a potência calculada pelos polinômios com a registrada pelo aerogerador, com análise de correlação ( $r$ ) e erro absoluto relativo (RAE), pois estamos interessados no menor erro absoluto. Embora calculado, o MAE não foi inserido na tabela pois não acrescentava informações significativas nessa análise. Os resultados obtidos foram organizados conforme a Tabela 1.

Após a seleção dos dados, obtivemos 8 dias sem dados, 5 dias com correlação abaixo de 60\%, 12 dias com correlação acima de $90 \%$ e 5 dias entre $80 \%$ e $90 \%$ de correlação. Constatouse que quanto maior o número de dados utilizados no ajuste de curva maior a correlação e menor o erro, porém esses dados devem ser consecutivos, exemplificados pelos dias 2, com correlação de $89 \%$ e 7 , com correlação de $-10 \%$, conforme ilustrado nas Figura 2 e Figura 3. 
Tabela 1 - Coluna 1 - dia do mês; coluna 2 - quantidade de dados utilizados em ordem decrescente; coluna 3 - correlação entre os dados coletados e os gerados por P1; coluna 4 - erro absoluto relativo de $\mathrm{P} 1$; coluna 5 - correlação entre os dados coletados e os gerados por P2; coluna 6 - erro absoluto relativo de P2; coluna 7 - correlação entre os dados coletados e os gerados por P3; coluna 8 - erro absoluto relativo de P3; coluna 9 - correlação entre os dados coletados e os gerados por P4; coluna 10 - Erro absoluto relativo de P4.

\begin{tabular}{|cccccccccc|}
\hline \multirow{2}{*}{ Dia } & \multirow{2}{*}{ Dados } & \multicolumn{2}{c}{ Polinômio 1 (P1) } & \multicolumn{2}{c}{ Polinômio 2 (P2) } & \multicolumn{2}{c|}{ Polinômio 3 (P3) } & \multicolumn{2}{c|}{ Polinômio 4 (P4) } \\
\cline { 3 - 9 } & & R & RAE & r & RAE & r & RAE & r & RAE \\
\hline 10 & 1436 & 0,92 & 0,40 & 0,92 & 0,40 & 0,92 & 0,40 & 0,92 & 0,40 \\
15 & 1310 & 0,95 & 0,31 & 0,95 & 0,31 & 0,95 & 0,31 & 0,95 & 0,31 \\
18 & 1135 & 0,91 & 0,40 & 0,91 & 0,40 & 0,91 & 0,40 & 0,91 & 0,40 \\
16 & 1118 & 0,94 & 0,42 & 0,94 & 0,41 & 0,94 & 0,43 & 0,94 & 0,41 \\
22 & 1111 & 0,97 & 0,28 & 0,97 & 0,27 & 0,97 & 0,29 & 0,97 & 0,28 \\
9 & 1026 & 0,92 & 0,39 & 0,92 & 0,39 & 0,92 & 0,39 & 0,92 & 0,39 \\
19 & 1025 & 0,91 & 0,39 & 0,91 & 0,39 & 0,91 & 0,39 & 0,91 & 0,39 \\
23 & 961 & 0,96 & 0,33 & 0,96 & 0,33 & 0,96 & 0,34 & 0,96 & 0,33 \\
17 & 906 & 0,89 & 0,50 & 0,89 & 0,49 & 0,89 & 0,51 & 0,89 & 0,49 \\
26 & 638 & 0,93 & 0,60 & 0,93 & 0,58 & 0,93 & 0,63 & 0,93 & 0,58 \\
25 & 595 & 0,95 & 0,47 & 0,95 & 0,46 & 0,95 & 0,50 & 0,95 & 0,46 \\
5 & 589 & 0,86 & 0,65 & 0,86 & 0,65 & 0,86 & 0,66 & 0,86 & 0,65 \\
21 & 575 & 0,97 & 0,24 & 0,97 & 0,24 & 0,97 & 0,25 & 0,97 & 0,24 \\
24 & 538 & 0,89 & 1,05 & 0,89 & 1,02 & 0,89 & 1,13 & 0,89 & 1,02 \\
11 & 488 & 0,81 & 0,61 & 0,81 & 0,61 & 0,81 & 0,62 & 0,81 & 0,61 \\
7 & 355 & $-0,10$ & 1,23 & $-0,10$ & 1,23 & $-0,10$ & 1,23 & $-0,10$ & 1,23 \\
2 & 343 & 0,89 & 0,52 & 0,89 & 0,51 & 0,89 & 0,54 & 0,89 & 0,51 \\
3 & 292 & 0,96 & 0,27 & 0,96 & 0,26 & 0,96 & 0,27 & 0,96 & 0,26 \\
27 & 249 & 0,03 & 4,52 & 0,03 & 4,52 & 0,03 & 4,53 & 0,03 & 4,52 \\
29 & 137 & $-0,16$ & 1,18 & $-0,16$ & 1,17 & $-0,16$ & 1,19 & $-0,16$ & 1,18 \\
1 & 62 & 0,56 & 1,01 & 0,56 & 1,00 & 0,56 & 1,05 & 0,56 & 1,00 \\
6 & 40 & $-0,04$ & 1,04 & $-0,04$ & 1,04 & $-0,04$ & 1,06 & $-0,04$ & 1,04 \\
\hline
\end{tabular}

Fonte: Dados da pesquisa (2019).

Figura 2 - Registro de potência inserida na rede ao longo do dia 2 de junho de 2017 pelo aerogerador. Em destaque, os instantes em que foram registradas intensidades das velocidades do vento maior que $3 \mathrm{~m} / \mathrm{s}$ e em que a potência foi maior que zero, dados estes utilizados no ajuste de curva.

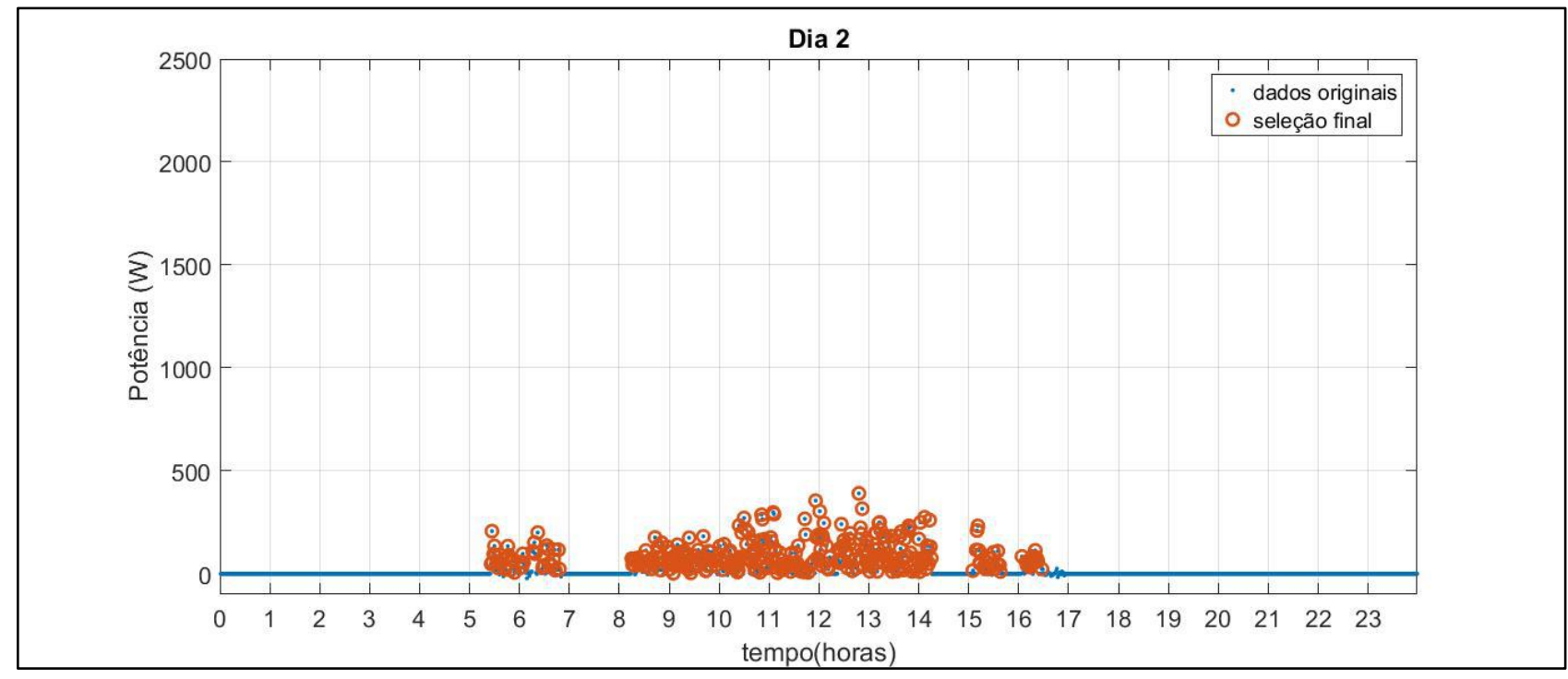

Fonte: Dados da pesquisa (2019). 
Figura 3 - Registro de potência inserida na rede ao longo do dia 7 de junho de 2017 pelo aerogerador. Em destaque, os instantes em que foram registradas intensidades das velocidades do vento maior que $3 \mathrm{~m} / \mathrm{s}$ e em que a potência foi maior que zero, dados estes utilizados no ajuste de curva.

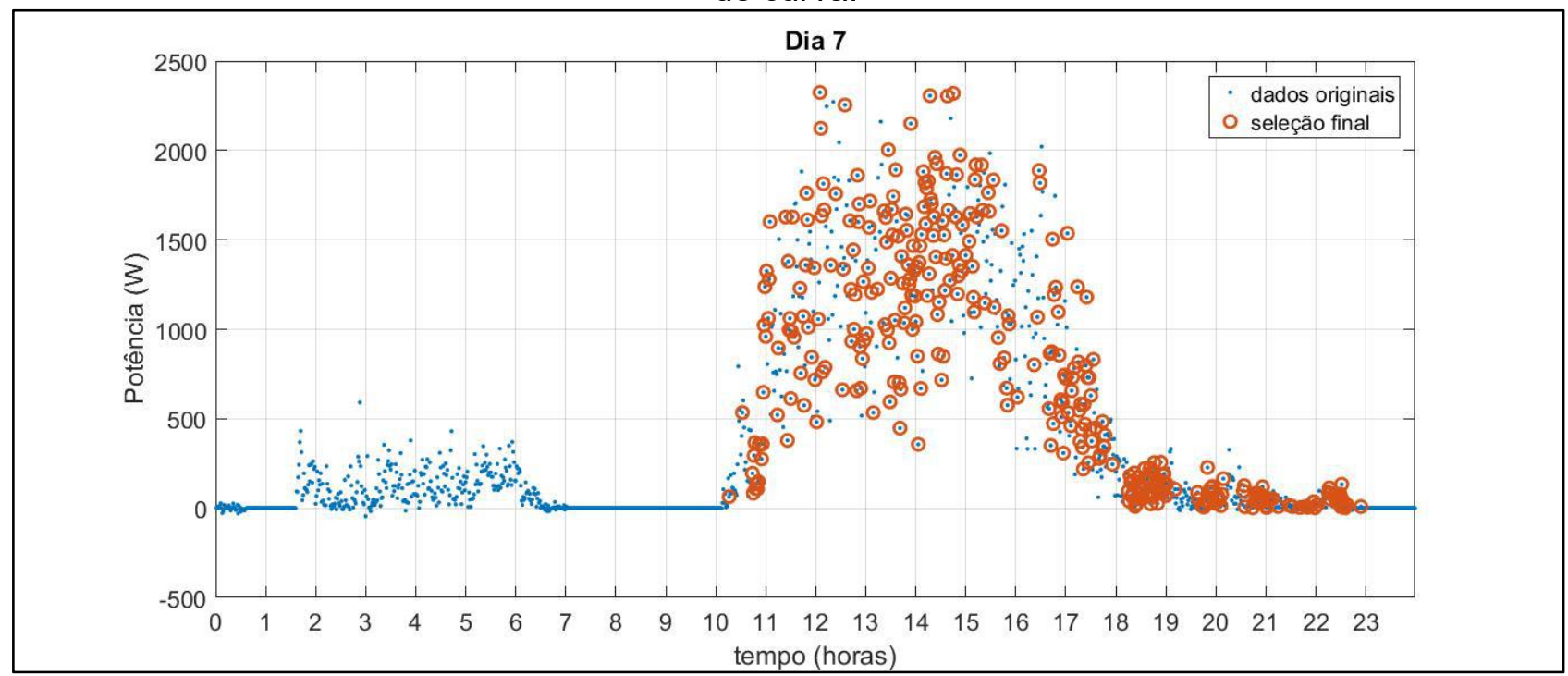

Fonte: Dados da pesquisa (2019).

No dia 2, conforme mostra a Figura 2, há uma quantidade menor de dados, porém em intervalos consecutivos; já no dia 7 , conforme mostra a Figura 3 , os dados utilizados são espaçados, o que explicaria a baixa correlação, já que os resultados do modelo de ajuste por mínimos quadrados dependem do número de dados usados para sua representação.

Outra explicação para esse erro é que os dados registrados fora da curva na Figura 1 são do dia 7, explicitado na Figura 4. Além disso, por meio da análise do erro verificou-se que os polinômios obtidos são bons preditores.

A Figura 4 mostra os registros dos dias 7, 27, 29 e 6, em que a correlação com a curva é baixa, dias estes em que a potência injetada na rede estava fora do padrão. Outra diferença entre os dias 2 e 7 é que a potência registrada no dia 7 é maior que no dia 2, porém no dia 10, com correlação de $92 \%$, a potência registrada também é alta, como mostra a Figura 5. 
Figura 4 - Dados originais registrados no mês de junho 2017. Em destaque, os dados selecionados nos dias 7 (o) em vermelho, $27\left(^{*}\right)$ em amarelo, $29(\mathrm{x})$ em ciano e $6(+)$ em verde nos quais obtivemos menores correlações e maiores erros.

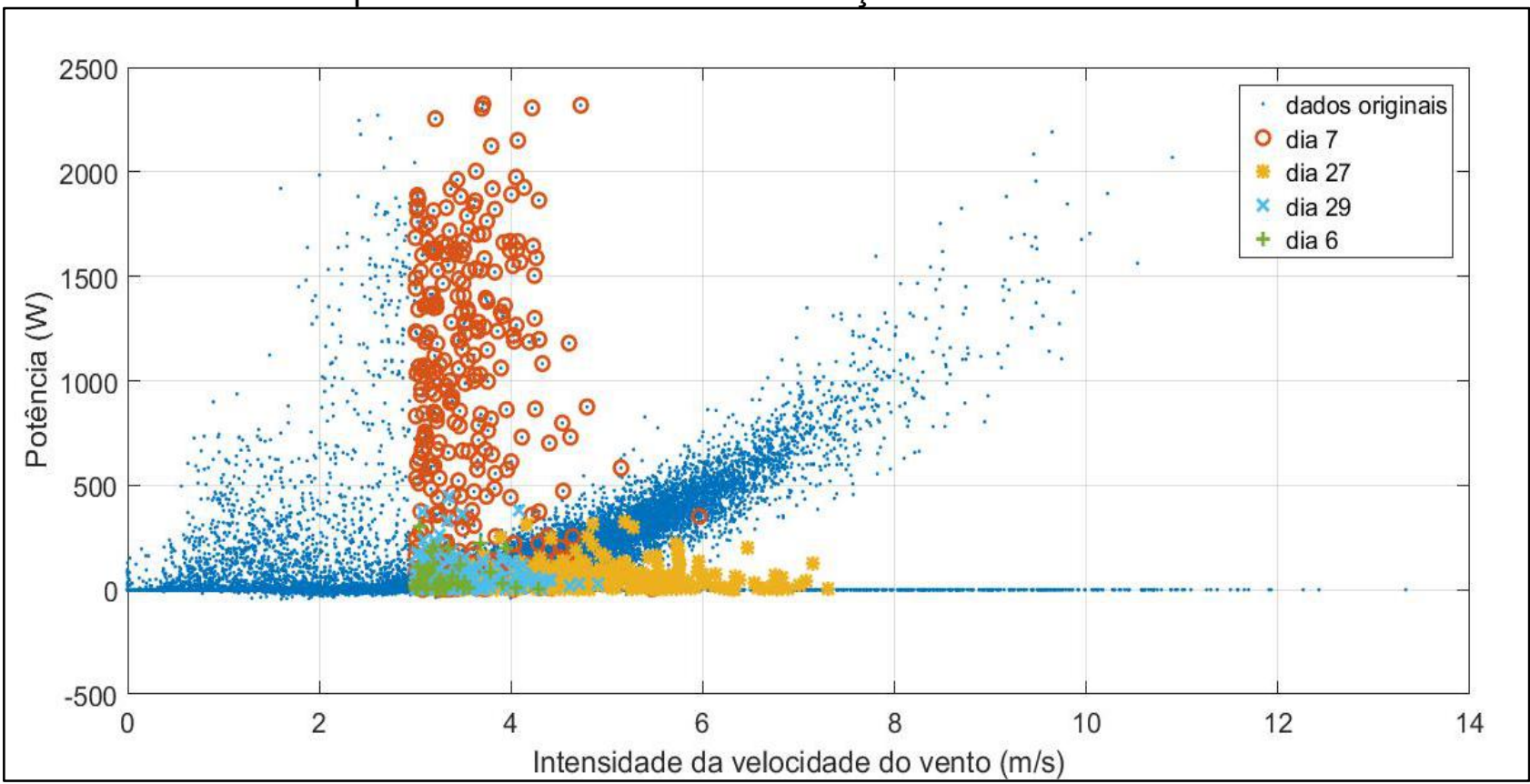

Fonte: Dados da pesquisa (2019).

Figura 5 - Registro de potência inserida na rede ao longo do dia 10 de junho de 2017 pelo aerogerador. Em destaque, os instantes em que foram registradas intensidades das velocidades do vento maior que $3 \mathrm{~m} / \mathrm{s}$ e em que a potência foi maior que zero, dados estes utilizados no ajuste de curva.

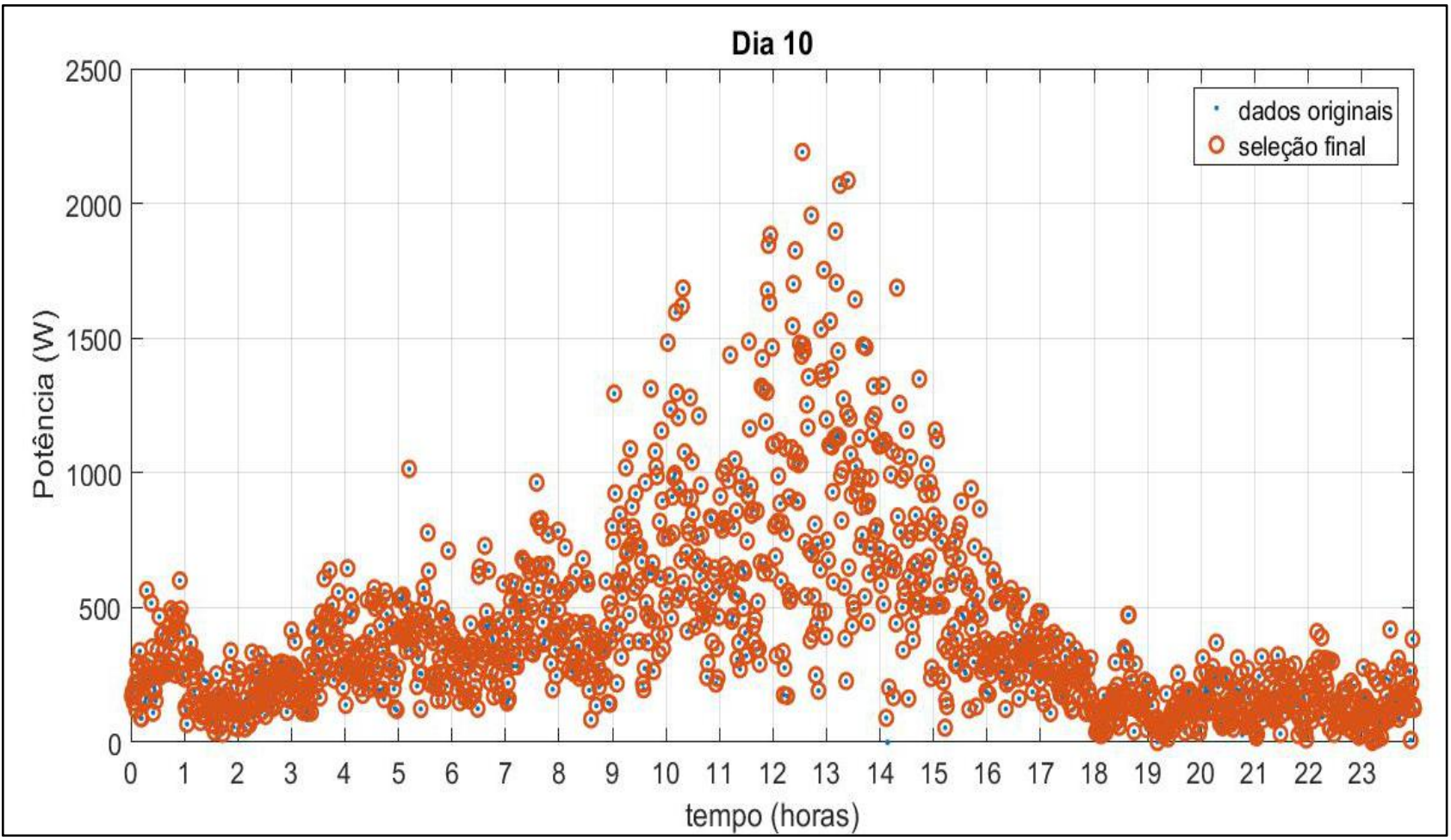

Fonte: Dados da pesquisa (2019). 
Figura 6 - Registro de potência inserida na rede ao longo do dia 22 de junho de 2017 pelo aerogerador. Em destaque, os instantes em que foram registradas intensidades das velocidades do vento maior que $3 \mathrm{~m} / \mathrm{s}$ e em que a potência foi maior que zero, dados estes utilizados no ajuste de curva.

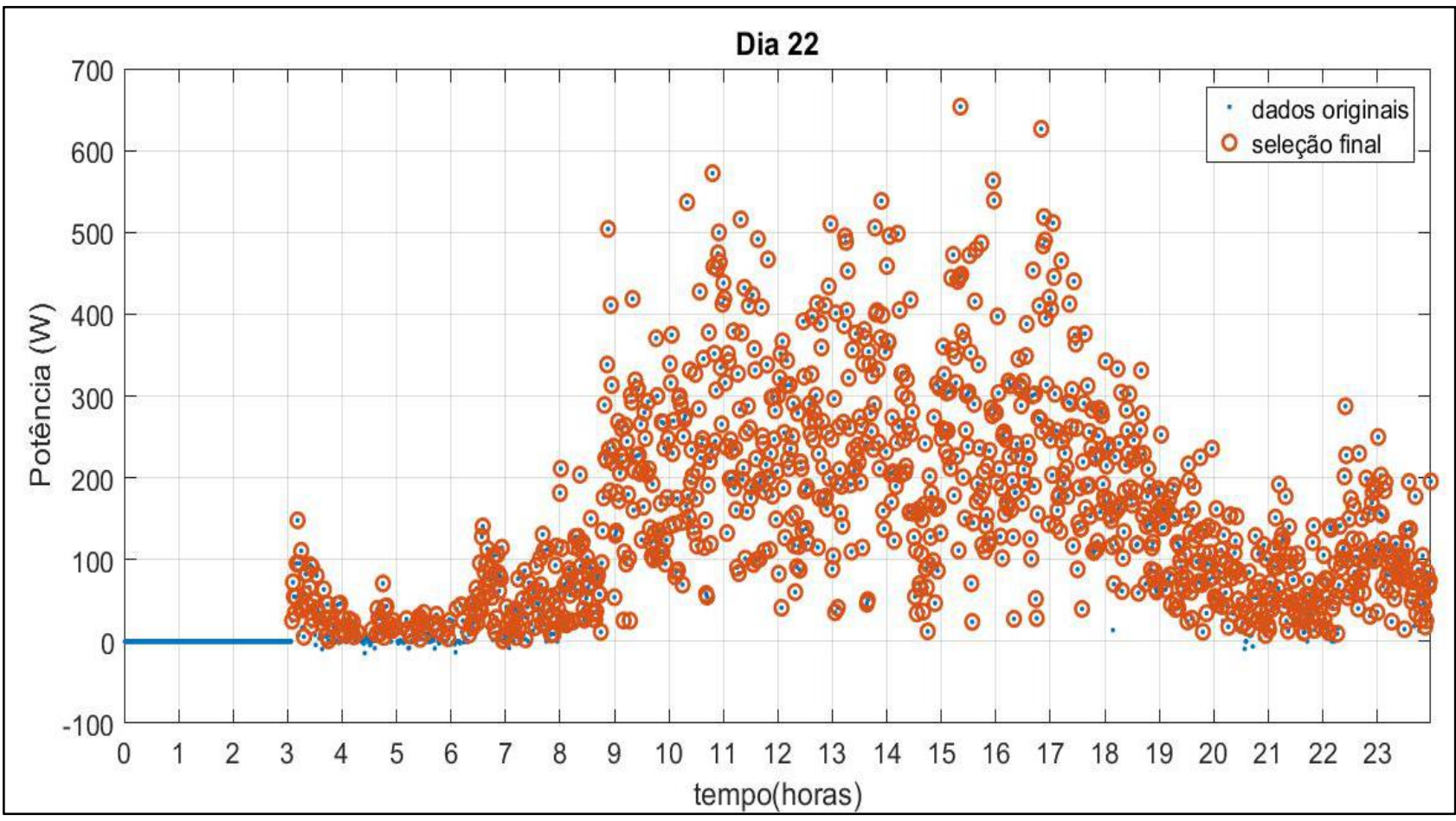

Fonte: Dados da pesquisa (2019).

Os resultados dos quatro diferentes polinômios são muito similares, porém os menores erros são de P2.

Com o auxílio do software matemático obtivemos o seguinte polinômio:

$$
P 5=-2,7489 . v^{3}+79,4905 \cdot v^{2}-426,1495 \cdot v+730,9483
$$

Ao fazer a análise estatística entre P2 (Método dos Mínimos Quadrados) e P5 (construído com auxílio do software), comparando com os valores coletados no mês de junho, encontrou-se a correlação entre os dados originais e P2 maior ou igual a P5, e os erros de P5 maiores que de P2, com exceção do erro médio absoluto no dia 10, mostrados na Tabela 2.

Tabela 2 - Coluna 1 - dia do mês; coluna 2 - número de dados utilizados; coluna 3 - correlação entre os dados coletados e os gerados por P2; coluna 4 - correlação entre os dados coletados e os gerados por P5; coluna 5 - erro absoluto relativo de P2; coluna 6 - erro absoluto relativo de P5; coluna 7 - erro médio absoluto de P2; coluna 8 - erro médio absoluto de P5.

\begin{tabular}{|c|c|c|c|c|c|c|c|}
\hline \multirow{2}{*}{ Dia } & \multirow{2}{*}{ Dados } & \multicolumn{2}{|c}{ R } & \multicolumn{2}{c|}{ RAE } & \multicolumn{2}{c|}{ MAE } \\
\cline { 3 - 8 } & & P2 & P5 & P2 & P5 & P2 & P5 \\
\hline 10 & 1436 & 0,92 & 0,92 & 0,4 & 0,38 & 99,94 & 96,41 \\
\hline 15 & 1310 & 0,95 & 0,95 & 0,31 & 0,32 & 36,96 & 37,65 \\
\hline 18 & 1135 & 0,91 & 0,91 & 0,4 & 0,42 & 54,82 & 57,67 \\
\hline 16 & 1118 & 0,94 & 0,93 & 0,41 & 0,51 & 34,02 & 42,29 \\
\hline 22 & 1111 & 0,97 & 0,96 & 0,27 & 0,34 & 27,37 & 34,33 \\
\hline 9 & 1026 & 0,92 & 0,92 & 0,39 & 0,4 & 59,35 & 61,66 \\
\hline 19 & 1025 & 0,91 & 0,91 & 0,39 & 0,4 & 64,01 & 65,27 \\
\hline
\end{tabular}




\begin{tabular}{|c|c|c|c|c|c|c|c|}
\hline 23 & 961 & 0,96 & 0,95 & 0,33 & 0,37 & 30,74 & 34,85 \\
\hline 17 & 906 & 0,89 & 0,88 & 0,49 & 0,57 & 43,48 & 50,24 \\
\hline 26 & 638 & 0,93 & 0,91 & 0,58 & 0,83 & 31,11 & 44,25 \\
\hline 25 & 595 & 0,95 & 0,93 & 0,46 & 0,62 & 27 & 36,57 \\
\hline 5 & 589 & 0,86 & 0,85 & 0,65 & 0,74 & 51,14 & 58,9 \\
\hline 21 & 575 & 0,97 & 0,97 & 0,24 & 0,27 & 30,87 & 34,33 \\
\hline 24 & 538 & 0,89 & 0,86 & 1,02 & 1,66 & 32,92 & 53,73 \\
\hline 11 & 488 & 0,81 & 0,8 & 0,61 & 0,66 & 46,34 & 50,44 \\
\hline 7 & 355 & $-0,1$ & $-0,11$ & 1,23 & 1,21 & 711,55 & 698,08 \\
\hline 2 & 343 & 0,89 & 0,87 & 0,51 & 0,75 & 27,08 & 39,92 \\
\hline 3 & 292 & 0,96 & 0,96 & 0,26 & 0,31 & 35,28 & 41 \\
\hline 27 & 249 & 0,03 & 0,01 & 4,52 & 4,57 & 193,95 & 196,41 \\
\hline 1 & 137 & $-0,16$ & $-0,14$ & 1,17 & 1,23 & 71,8 & 75,24 \\
\hline 6 & 62 & 0,56 & 0,49 & 1 & 1,3 & 40,79 & 53,29 \\
\hline
\end{tabular}

Fonte: Dados da pesquisa (2019).

A Figura 7 mostra os dados de junho de 2017, utilizados neste estudo, comparados com a curva de potência do aerogerador com registro de potência injetada na rede de setembro de 2016 a junho de 2017 (GEPOC, 2017) e as curvas geradas por P2 e por P5. Observa-se que a curva gerada por P2 é válida até ventos com intensidades até $10 \mathrm{~m} / \mathrm{s}$, que é próxima à potência nominal (aproximadamente 2kW). A partir de $11 \mathrm{~m} / \mathrm{s}$, P5 apresenta maiores erros com ventos de menor intensidade, mas se aproxima mais da curva oficial (fornecida pelo fabricante).

Figura 7 - Dados coletados pelo aerogerador em junho de 2017 (.), curva de potência do aerogerador estudado (GEPOC, 2017) (+), curva de P2 (--) e curva de P5 (.-).

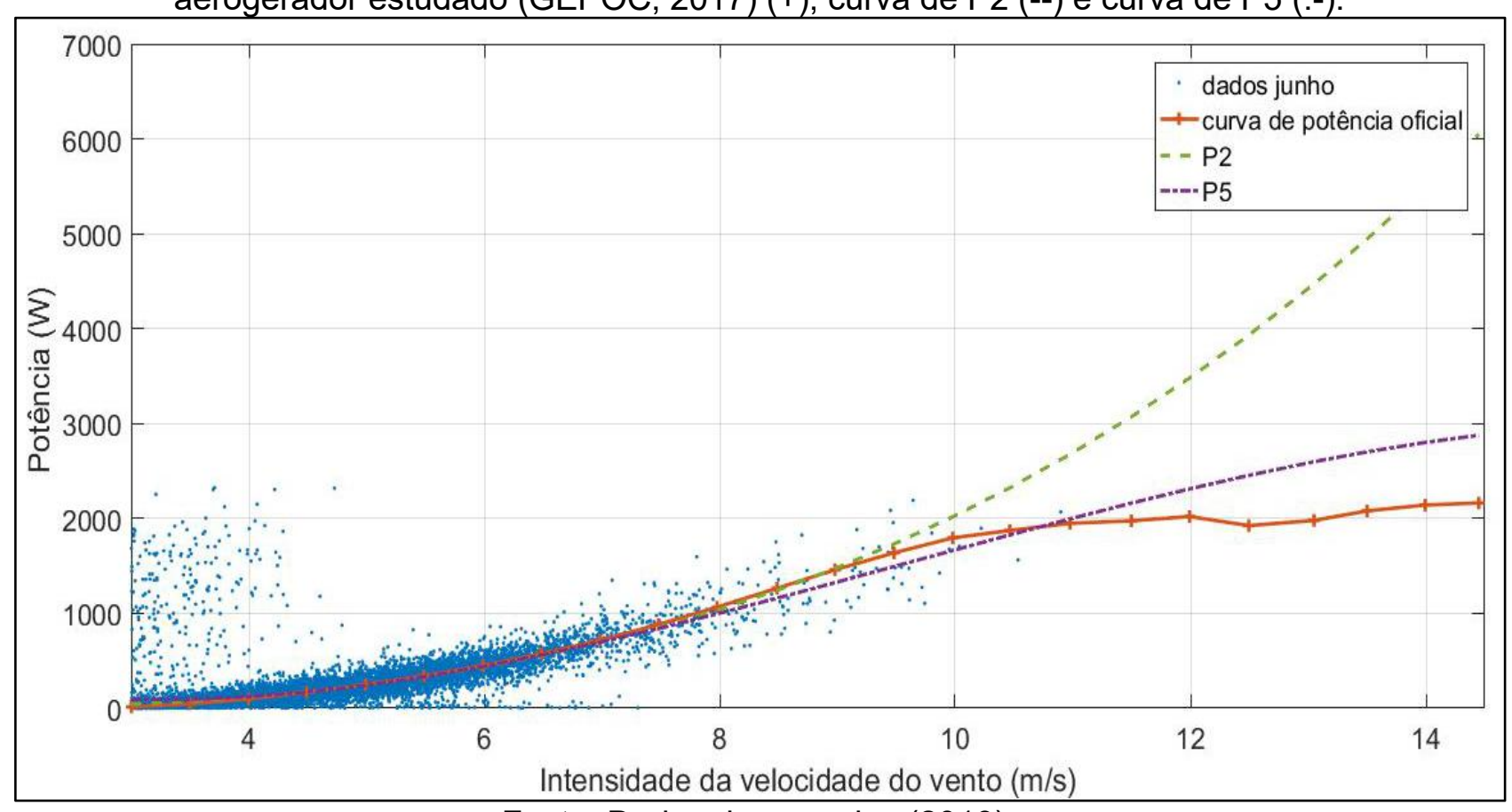

Fonte: Dados da pesquisa (2019). 
A Tabela 3 compara P2 e P5 com a curva oficial de potência (GEPOC, 2017), apresentada na Figura 7, mostrando que, para velocidades até $9 \mathrm{~m} / \mathrm{s}$, o polinômio com menores erros é o construído manualmente (P2), porém com velocidades altas esse polinômio apresenta maiores erros e menor correlação; ou seja, com velocidades entre $9 \mathrm{~m} / \mathrm{s}$ e $11 \mathrm{~m} / \mathrm{s}$, nas quais o aerogerador entra em potência nominal (constante), esse polinômio não mostra os melhores resultados.

Tabela 3 - Coluna 1 - intervalo de intensidade das velocidades do vento analisadas; coluna 2 polinômio analisado; coluna 3 - correlação entre a curva de potência do aerogerador e os gerados pelos polinômios; coluna 4 - erro absoluto relativo entre a curva de potência do aerogerador e a gerada pelos polinômios; coluna 5 - erro médio absoluto entre a curva de potência do aerogerador e a gerada pelos polinômios.

\begin{tabular}{|c|c|c|c|c|}
\hline & & r & RAE & MAE \\
\hline $\begin{array}{c}\text { Vento com velocidades de } \\
\mathbf{3} \text { a 14,5 m/s }\end{array}$ & P2 & 0,89 & 1,02 & 757,34 \\
\hline $\begin{array}{c}\text { Vento com velocidades de } \\
\mathbf{3} \text { a 9 m/s }\end{array}$ & P5 & 0,97 & 0,25 & 187,11 \\
\cline { 2 - 5 } & P2 & 1 & 0,05 & 20,18 \\
\hline
\end{tabular}

Fonte: Dados da pesquisa (2019).

\section{Considerações Finais}

O trabalho mostra que há diferenças entre a aplicação do Método dos Mínimos Quadrados para a modelagem da potência gerada pelo aerogerador de pequeno porte e a solução utilizando polinômios interpoladores do software Octave. O polinômio construído pelo Método dos Mínimos Quadrados apresentou menores erros e maiores correlações com os dados diários e, ao comparálos com a curva de potência, este é válido para intensidade do vento até $10 \mathrm{~m} / \mathrm{s}$, com os melhores resultados até $9 \mathrm{~m} / \mathrm{s}$. Para valores superiores a $11 \mathrm{~m} / \mathrm{s}$, o aerogerador entra em potência nominal (constante a $2 \mathrm{~kW}$ ), e essas diferenças podem ser explicadas a partir do método utilizado.

Conceitualmente, para cada $x_{k}$ deveria existir um $y_{k}$. Ocorre que nos dados gerados pelo aerogerador, por vezes, são fornecidos mais de um $y_{k}$. Tentamos introduzir uma variável $z_{k} \mathrm{e}$ estender o ajuste para uma $y_{k}=f\left(x_{k}, z_{k}\right)$, mas os resultados não se mostraram satisfatórios. Ao comparar com os dados de teste, nos quais essas repetições também ocorrem, nossa estratégia se revelou mais adequada, visto que as correlações foram melhores e, dessa forma, os objetivos do trabalho foram atingidos.

\section{Agradecimentos}

Este trabalho foi possível graças ao afastamento concedido pelo Instituto Federal de Educação, Ciência e Tecnologia do Rio Grande do Sul (IFRS), para qualificação. 


\section{Referências}

BRASIL. Ministério das Minas e Energia. Plano Nacional de Energia 2030. 2007. Disponível em: http://www.mme.gov.br/web/guest/publicacoes-e-indicadores/plano-nacional-de-energia-2050.

Acesso em: 11 set. 2019.

DUFFIE, N. A.; YANG, S. M.; BOLLINGER, J. G. Generation of Parametric Kinematic ErrorCorrection Functions from Volumetric Error Measurements. CIRP Annals. v. 34, n. 1, p. 435-438, 1985. DOI: https://doi.org/10.1016/S0007-8506(07)61806-X.

GEPOC. Grupo de Eletrônica de Potência e Controle. Santa Maria. 2017. Disponível em: http://eolicagepoc.ct.ufsm.br/laete/. Acesso em: 27 dez. 2019.

KASSEM, Y; HÜSEYIN, Ç; ABDELRAHMAN, A. Evaluation of Wind Energy Potential and Economic Analysis of Wind Energy Turbine Using Present Value Cost Method at Famagusta, Rizokarpaso, Kyrenia, Morphou, Nicosia and Ercan in Cyprus: Case Study. Conference FullPaper Proceedings Book, Cyprus Science University, p. 63-80, dez. 2017.

LOPES, V. L. R. Cálculo Numérico: Aspectos Teóricos e Computacionais. 2.ed. São Paulo: Pearson, 2008.

MATSUMOTO, E. Y. Matlab 6: Fundamentos de Programação. São Paulo: Érica, 2001.

PINTO, L. I. C.; LIMA, F. J. L. de; MARTINS, F. R.; PEREIRA, E. B. Testes de Sensibilidade para Diferentes Parametrizações Cumulus do Modelo Wrf para Melhorar as Estimativas de Vento. In: CONGRESSO BRASILEIRO DE ENERGIA SOLAR, 7., Gramado, 2018. Anais... Associação Brasileira de Energia Solar, Gramado, 2018.

TRANCOSO, A. R.; DOMINGOS, D.; PESTANA, R. Previsibilidade da Energia Eólica. In: JORNADAS CIENTIIFICAS DE LA ASOCIACIÓN METEOROLÓGICA ESPAÑOLA APLICACIONES METEOROLÓGICAS, 29., ENCUENTRO HISPANO-LUSO DE METEOROLOGÍA METEOROLOGÍA Y EFICIENCIA ENERGÉTICA, 7., abr. 2006, Pamplona, Espanha. 29a Jornadas Científicas de la AME (2006). Madri: Asociación Meteorológica Española, p. 1-9, 2006. Disponível em: http://hdl.handle.net/20.500.11765/5138. Acesso em: 27 dez. 2019. 